\title{
Pacific Conference on Spectroscopy and Dynamics
}

\section{Virtual Special Issue}

Heterogeneous Interactions between

Gas-Phase Pyruvic Acid and

Hydroxylated Silica Surfaces: A

Combined Experimental and

Theoretical Study

Oxidation of a Molecule by the

Biexcitonic State of a CdS Quantum

Dot

Nonadiabatic Investigation of the

Electronic Spectroscopy of trans-1,3-

Butadiene

UV-Vis Action Spectroscopy of

Guanine, 9-Methylguanine, and

Guanosine Cation Radicals in the

Gas Phase

Modeling Carbon Dioxide

Vibrational Frequencies in Ionic

Liquids: IV. Temperature

Dependence

Absorption Spectra of Acetylene,

Vinylidene, and Their Deuterated

Isotopologues on $\mathrm{Ab}$ Initio Potential

Energy and Dipole Moment Surfaces

Nonstatistical Dissociation Dynamics of Nitroaromatic Chromophores
Jing Chang, Lifen Guo, Ruifang Wang, Jie

Statistical Analysis of the Effect of

Deuteration on Quantum

Delocalization in $\mathrm{CH}_{5}^{+}$

First-Principles Calculation of Jahn-

Teller Rotational Distortion

Parameters

Effects of Intra- and Intermolecular

Hydrogen Bonding on O-H Bond

Photodissociation Pathways of a

Catechol Derivative
Yuan Fang, Dominika Lesnicki, Kristin J.

Wall, Marie-Pierre Gaigeot, Marialore

Sulpizi, Veronica Vaida, Vicki H. Grassian

Shichen Lian, Joseph A. Christensen,

Mohamad S. Kodaimati, Cameron R.

Rogers, Michael R. Wasielewski, Emily A.

Weiss

Scott M. Rabidoux, Robert J. Cave, John F.

Stanton

Andy Dang, Yue Liu, František Tureček

10.1021/acs.jpca.9b01542

Clyde A. Daly, Cecelia Allison, Steven A.

Corcelli

Mou, Haisheng Ren, Jianyi Ma, Hua Guo

K. Jacob Blackshaw, Belinda I. Ortega,

Naa-Kwarley Quartey, Wade E. Fritzeen,

Robert T. Korb, Annalise K. Ajmani,

Lehman Montgomery, Marcus Marracci,

Geronimo Gudino Vanegas, John Galvan,

Zach Sarvas, Andrew S. Petit, Nathanael M.

Kidwell

10.1021/acs.jpca.8b10224

10.1021/acs.jpcc.9b00210

10.1021/acs.jpca.9b01021

10.1021/acs.jpcb.9b01863

10.1021/acs.jpca.9b02662

10.1021/acs.jpca.9b02312

Meredith E. Fore, Anne B. McCoy

10.1021/acs.jpca.9b02685

Ketan Sharma, Scott Garner, Terry A.

Miller, John F. Stanton

Christopher Grieco, Alex T. Hanes, Lluís

Blancafort, Bern Kohler 10.1021/acs.jpca.9b04573

10.1021/acs.jpca.9b03360 
Screening Links Transport and

Recombination Mechanisms in Lead

Halide Perovskites

Organic Enrichment at Aqueous

Interfaces: Cooperative Adsorption

of Glucuronic Acid to DPPC

Monolayers Studied with Vibrational

Sum Frequency Generation

Resonance Hyper-Raman Scattering

from CdSe and CdS Nanocrystals

Kinetic Investigations of the $\mathrm{CH}$

$\left(\mathrm{X}^{2} \Pi\right)$ Radical Reaction with

Cyclopentadiene

Length and Charge of Water-Soluble

Peptoids Impact Binding to

Phospholipid Membranes

Specifics about Specific Ion

Adsorption from Heterodyne-

Detected Second Harmonic

Generation

Tests of the Stokes-Einstein Relation through the Shear Viscosity

Activation Energy of Water

Conformational Study of the Jet-

Cooled Diketopiperazine Peptide

Cyclo Tyrosyl-Prolyl

State-Selective Polariton to Dark

State Relaxation Dynamics

Raman and Quantum Yield Studies of Trp48- $d_{5}$ in Azurin: Closed-Shell and Neutral Radical Species

Exciton Localization and Radiative

Lifetimes in CdSe Nanoplatelets

Effect of Ions on the Optical

Absorption Spectra of Aqueously

Solvated Chromophores

Decay-Associated Fourier

Spectroscopy: Visible to Shortwave

Infrared Time-Resolved

Photoluminescence Spectra

Interpretation of the $\mathrm{THz}-\mathrm{THz}-$

Raman Spectrum of Bromoform
Casey L. Kennedy, Andrew H. Hill, Erik M. 10.1021/acs.jpcc.9b03235

Grumstrup

Katie A. Link, Gabrielle N. Spurzem,

Aashish Tuladhar, Zizwe Chase, Zheming

Wang, Hongfei Wang, Robert A. Walker

Rui Tan, David F. Kelley, Anne Myers

Kelley

Kacee L. Caster, Zachery N. Donnellan,

Talitha M. Selby, F. Goulay

Madeleine R. Landry, Jacenda L. Rangel,

Vivian P. Dao, Morgan A. MacKenzie,

Fabiola L. Gutierrez, Kalli M. Dowell, Anna

L. Calkins, Amelia A. Fuller, Grace Y.

Stokes

Mavis D. Boamah, Paul E. Ohno, Emilie

Lozier, Jacqueline Van Ardenne, Franz M.

Geiger

10.1021/acs.jpca.9b02255

10.1021/acs.jpcc.9b04645

10.1021/acs.jpca.9b03813

10.1021/acs.jpcb.9b04641

10.1021/acs.jpcb.9b04425
Camina H. Mendis, Zeke A. Piskulich,

Ward H. Thompson

Ariel Pérez-Mellor, Ivan Alata, Valeria

Lepère, Anne Zehnacker

Lepere, An Zehneck

Bo Xiang, Raphael F. Ribeiro, Liying Chen, 10.1021/acs.jpca.9b04601

Jiaxi Wang, Matthew Du, Joel Yuen-Zhou,

Wei Xiong

Joel J. Rivera, Justine H. Liang, Gregory R.

Shimamura, Hannah S. Shafaat, Judy E.

Kim

David P. Morgan, David F. Kelley

10.1021/acs.jpcc.9b04639

Sapana V. Shedge, Tim J. Zuehlsdorff,

Michael J. Servis, Aurora E. Clark,

Christine M. Isborn

Timothy L. Atallah, Anthony V. Sica,

Ashley J. Shin, Hannah C. Friedman, Yaniv

K. Kahrobai, Justin R. Caram

Ioan B. Magdău, Griffin J. Mead, Geoffrey

10.1021/acs.jpca.9b05165

10.1021/acs.jpcb.9b04647

10.1021/acs.jpcb.9b04529

10.1021/acs.jpcb.9b04655

10.1021/acs.jpca.9b03163

10.1021/acs.jpca.9b04924

A. Blake, Thomas F. Miller 